Volume 107, Number 3

tion in clinical lung transplantation. J THORAC CARDIOVASC SURG 1993;105:247-52.

5. Schäfers H-J, Haverich A, Wagner TOF, Wahlers T, Alken A, Borst HG. Decreased incidence of bronchial complications following lung transplantation Eur J Cardiothorac Surg 1992;6:174-9.

\section{Aortoinnominopexy versus innominate artery reimplantation for displaced innominate artery}

\section{To the Editor:}

Displacement of the innominate artery can cause tracheal compression and produce such problems as reflex apnea, respiratory distress, and recurrent upper respiratory tract infections. Reported prevalences of displaced innominate artery vary widely. Institutional awareness and aggressiveness on the part of the otolaryngologist leads to increased recognition and higher prevalence figures. The main diagnostic tool is bronchoscopy, which shows the characteristic anterior pulsatile compression of the trachea.

Because the tracheal rings are soft and collapsible in infancy, symptoms usually appear early. The condition of the patient, however, gradually improves, and rarely do significant problems persist beyond infancy. Selecting patients for surgical correction can be difficult. Reflex apnea, however, can be life-threatening, and probably patients with such problems should be operated on provided results of neurologic, sleep, and reflex studies are normal.

Which surgical approach is most appropriate for displaced innominate artery is not settled. Hawkins, Bailey, and Clark ${ }^{1}$ reported that transection and reimplantation produced resolution of symptoms in $97 \%$ of patients. In their response to the criticisms of Backer, Hollinger, and Mavroudis, ${ }^{2}$ they claimed that they switched to reimplantation because of two failures among nine patients undergoing suspension, with only $50 \%$ of patients having immediate relief of symptoms. The reimplantation procedure, however, appears radical for a problem that is self-limiting, and there are always long-term concerns with vascular anastomosis in infancy.

Simple suspension of the innominate artery is challenging because of lack of sturdy enough tissue attached to the artery to hold sutures. Because of such problems, materials such as muscles and pericardium had been used to loop around the innominate artery. We have also reported our extensive experience $^{3-6}$ with operations for displaced innominate artery in more than 70 patients, and in 1977 we even submitted our early series to The American Association for Thoracic Surgery. Our technique has evolved through the years from simple suspension to aortoinnominopexy. We found that the pericardium attached to the aortoinnominate junction is strong and holds stitches well. The procedure is performed through a short right thoracotomy incision. The right lobe of the thymus is mobilized, the pericardium is opened, and a strip is left at the base of the innominate artery (Fig. 1). Three U stitches with pledgets, placed parallel to each other through the pericardium, are passed through the sternal periosteum and tied over pledgets. This effectively pulls the innominate artery and distal ascending aorta away from the trachea. With the current technique, we have not seen disruption of the repair encountered with simple suspension.

Serafin Y. DeLeon, $M D$

Jose A. Quinones, $M D$

Roque Pifarré, $M D$

Departments of Cardiothoracic Surgery and Pediatrics Loyola University Medical Center Maywood, IL 60153

\section{REFERENCES}

1. Hawkins JA, Bailey WW, Clark SM. Innominate artery compression of the trachea: treatment by reimplantation of
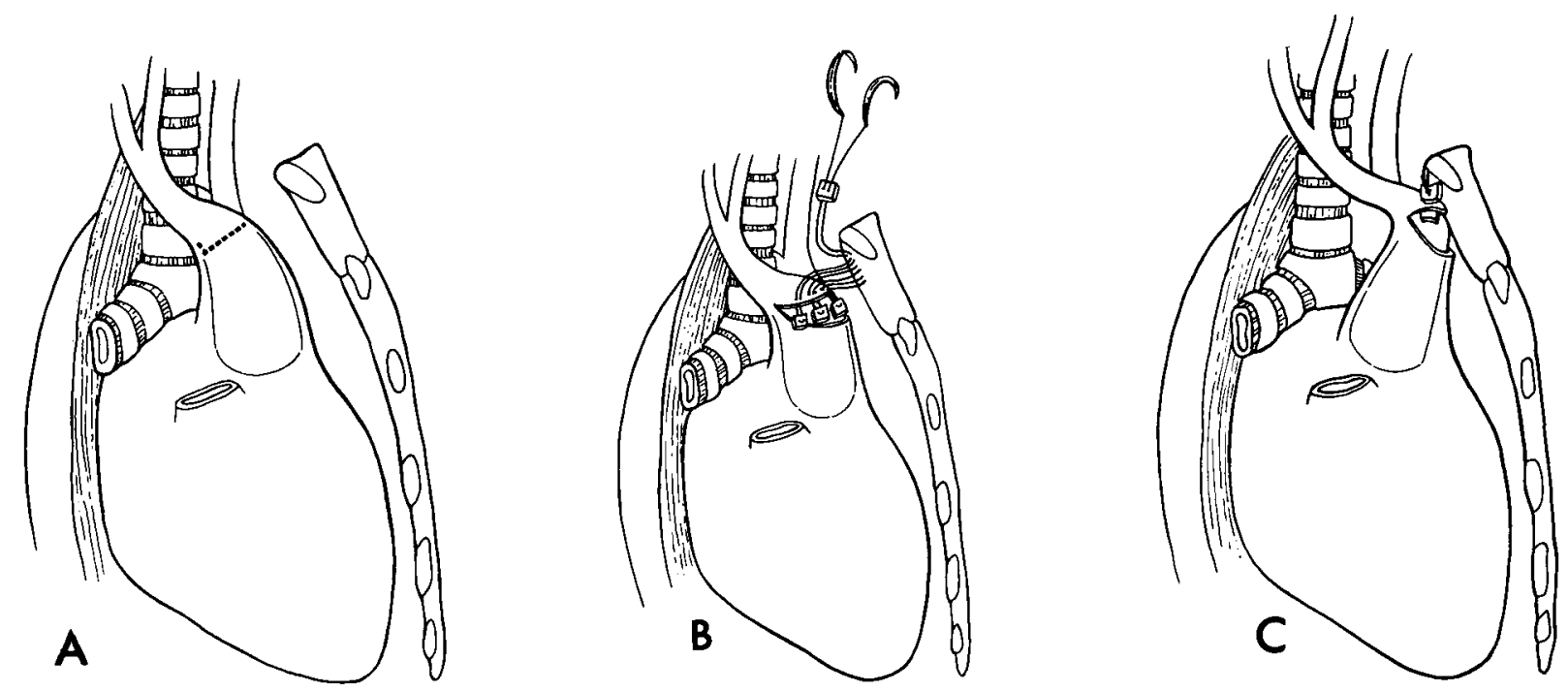

Fig. 1. A, Incision on the pericardium at the aortoinnominate junction is indicated. B, Three U stitches with pledgets are sutured into the sternal periosteum and tied over pledgets. $\mathbf{C}$, The aortoinnominate junction is pulled away from the trachea. 
the innominate artery. J THORAC CARDIOVASC SURG 1992; 103:678-82.

2. Backer CL, Holinger LD, Mavroudis C. Invited letter concerning: Innominate artery compression-division and reimplantation versus suspension. J THORAC CARDIOVASC SURG 1992;103:817-20.

3. DeLeon SY, Idriss FS, Nikaidoh H, Muster A, Tucker GF. Anterior tracheal compression by displaced innominate artery [Abstract]. Proc Inst Med 1977;31:320.

4. Idriss FS, Nikaidoh H, DeLeon SY, Koopot R. Surgery for vascular anomalies causing obstruction of the trachea and esophagus. In: Congenital heart disease. Tucker BL, Lindesmith GG, eds. New York: Grune \& Stratton, 1979:125-56.

5. Ardito JM, Ossoff RH, Tucker GF, DeLeon SY. Innominate artery compression of the trachea in infants with reflex apnea. Ann Otol Rhinol Laryngol 1980;89:401-5.

6. Backer CL, Ilbawi MN, Idriss FS, DeLeon SY. Vascular anomalies causing tracheoesophageal compression: review of experience in children. J THORAC CARDIOVASC SURG 1989; 98:725-31.

\section{Biologic vascular grafts in the venous position}

To the Editor:

The recent article by Ratto and colleagues ( $J$ THORAC CARDIOVASC SURG 1991;102:440-7) demonstrated a new application of heterologous connective tissue tube grafts (glutaraldehyde-treated, ovine grafts) as vascular substitutes in the venous system. Previously, good results after implantation of these grafts in animals were reported by Perloff and colleagues, ${ }^{1}$ but other investigators were not as successful. Although the graft itself is interesting and may be a step in the right direction toward finding a suitable material for venous replacement, the provocative findings of Ratto and colleagues raise some important concerns.

With light microscopy, Ratto and colleagues noted that the grafts were covered with a network of fibrin fibers, enmeshing platelets, and blood cells at 7 and 14 days after implantation. After only 2 more weeks, however, they suddenly noticed complete and regular endothelialization (day 28). Although the speed of endothelialization in venous grafts may be rapid because of good anchoring of endothelial cells in slow blood flow, their findings in only two grafts explanted at 1 month may not have been representative of the entire population. Because this is the most important period in the development of endothelialization, more observations may have been warranted. In addition, explanations between 14 and 28 days would have better defined how this rapid endothelialization may have occurred.

In the study of Ratto and colleagues, a staining method specific to endothelial cells would have identified the inner layer as endothelial. Peroxidase-antiperoxidase methods, which use a factor VIII-related surface antigen, are relatively easy to perform and have been used to correctly identify endothelial cells. This would have differentiated their findings of "completely and regularly endothelialized" connective tissue grafts from the expanded polytetrafluoroethylene graft neointima, which was "uniformly thinner than $200 \mu \mathrm{m}$ and ... completely lined with an endothelium-like layer."

Three possible sources of endothelialization have been suggested: (1) endothelial cell migration from the anastomosis, (2) circulating endothelial cells, and (3) migration of capillary endothelial cells through the graft from the perigraft tissue. In 1964 , Wesolowski and associates ${ }^{2}$ demonstrated that endothelialization from the anastomoses is slow, and it has also been shown that glutaraldehyde-treated biologic materials have a diminished affinity for cells on the luminal surface. More recently, Noishiki and coworkers ${ }^{3}$ demonstrated limited endothelialization (only 4 to $8 \mathrm{~mm}$ from the anastomoses) in heparinized, glutaraldehyde-treated ureteral grafts implanted into the canine inferior vena cava as long as 878 days after operation. The possibility of endothelial cell migration through the graft wall seems unlikely for similar reasons. Even though Ratto and colleagues implanted short grafts $(3.8 \mathrm{~cm})$ into the venous system of growing pigs, the source of the rapid endothelialization remains a mystery.

Ratto and colleagues did not discuss the cause of graft occlusion at 56 days in one of their collagen grafts. Was it intimal hyperplasia? Was it graft degeneration? Intimal hyperplasia is the main cause of graft occlusion in venous conduits in both the intermediate and long term. It therefore would not be surprising to see a short-term patency rate of $100 \%$ decrease significantly through the long term in the clinical setting. If the cause of graft occlusion was intimal hyperplasia or graft degeneration, this graft may not be as advantageous in clinical use, where long-term patency is the goal.

The report also raises some concerns with graft design and preimplantation treatment. Their Fig. 3 demonstrates multiple areas of thrombus deposition on the exposed Dacron polyester fibers only 1 hour after implantation. The origin of this phenomenon needs to be identified, but possible explanations can be suggested: (1) The graft was not fully matured or was injured during preparation. (2) The graft could not support the surrounding blood pressure adequately, even though it was in a low-pressure system. (3) The graft was extremely thrombogenic. Previous studies with similar grafts have also demonstrated important problems with connective tissue tube grafts implanted in to the canine arterial system. For example, endothelialization is limited to the anastomoses at 12 months, and thrombus formation is common on the luminal surface. ${ }^{4}$ In the study of Christie, Ketharanathan, and Perloff, 5 the cross-sectional area of the grafts increased during the long term; this may have been a result of their mesh framework with hexagonal interstices, which predisposed the grafts toward formation of pseudoaneurysms. Finally, glutaraldehyde-treated ovine connective tissue grafts have been found to be of limited usefulness because of bioincompatibility and degenerative changes. ${ }^{4}$

Glutaraldehyde treatment may not be as appropriate for the loosely arranged collagen fibers of a connective tissue tube as it is for biomaterials made of dense collagen fibers (heart valves and pericardium). Although dense connective tissue becomes strong after glutaraldehyde treatment because of the organization of its collagen fibrils, loose connective tissue tends to become rigid and brittle with glutaraldehyde treatment. In addition, glutaraldehyde is toxic and makes a biomaterial hydrophobic and bioincompatible. Alcohol-treatment may therefore be a better option to reduce antigenicity and maintain the graft's hydrophilic properties and cell affinity. In a study by Yamane, ${ }^{6}$ ethanol-treated bovine connective tissue tube grafts were implanted into the canine arterial system. Yamane demonstrated endothelial cells on the surface of the graft both extending from the anastomoses and appearing sporadically at the center of the graft 30 days after implantation. ${ }^{6}$ These findings suggest 